\title{
Graphene single-electron transistor as a spin sensor for magnetic adsorbates
}

\author{
J. W. González, ${ }^{1}$ F. Delgado, ${ }^{1}$ and J. Fernández-Rossier ${ }^{1,2}$ \\ ${ }^{1}$ International Iberian Nanotechnology Laboratory (INL), Av. Mestre José Veiga, 4715-330 Braga, Portugal \\ ${ }^{2}$ Departamento de Física Aplicada, Universidad de Alicante, 03690 San Vicente del Raspeig, Spain
}

(Received 11 December 2012; published 20 February 2013)

\begin{abstract}
We study single-electron transport through a graphene quantum dot with magnetic adsorbates. We focus on the relation between the spin order of the adsorbates and the linear conductance of the device. The electronic structure of the graphene dot with magnetic adsorbates is modeled through numerical diagonalization of a tight-binding model with an exchange potential. We consider several mechanisms by which the adsorbate magnetic state can influence transport in a single-electron transistor: tuning the addition energy, changing the tunneling rate, and in the case of spin-polarized electrodes, through magnetoresistive effects. Whereas the first mechanism is always present, the others require that the electrode has to have either an energy- or spin-dependent density of states. We find that graphene dots are optimal systems to detect the spin state of a few magnetic centers.
\end{abstract}

DOI: 10.1103/PhysRevB.87.085433

PACS number(s): 73.23.Hk, 81.05.ue, 85.75.Ss, 72.80.Vp

\section{INTRODUCTION}

Graphene is a very promising candidate for high-precision molecular sensing due to its extremely large surface to volume ratio and its electrically tunable large conductivity. ${ }^{1-3}$ On the other hand, being a zero-gap semiconductor with small mass and small density of spinfull nuclei makes graphene a material with potentially large spin lifetime for both carriers and host magnetic dopants. ${ }^{4}$ Taken together, these two ideas naturally lead to the use of graphene as a detector of the spin state of extrinsic magnetic centers in the form of magnetic adatoms, vacancies, and spinfull molecules. This connects with recently reported ${ }^{2,5}$ experiments in which gated graphene nanoconstrictions, operating in the single-electron transport (SET) regime, showed hysteresis in the linear conductance when a magnetic field is ramped. This behavior was observed both when the molecular magnets were intentionally deposited on graphene, as well as in carbon nanotubes, ${ }^{6}$ but also in the case of bare graphene nanojunctions ${ }^{5}$ where some type of graphene local moments ${ }^{7-9}$ are probably playing a role.

The graphene spin sensor experiments of Ref. 2 are performed in the Coulomb blockade regime, showing a vanishing linear conductance except in the neighborhood of specific values of the gate voltage $V_{g}$. This means that the graphene nanoconstriction is weakly coupled to the electrodes and has a charging energy larger than the thermal energy ( $T \sim 100 \mathrm{mK}$ ). The height of the linear conductance peaks is significantly smaller than $G_{0}=2 e^{2} / h$, the quantum of conductance. These conditions imply that transport takes place in the sequential regime. ${ }^{10}$ Thus current flow takes place due to sequential tunneling of electrons through the graphene constriction, which we refer to as the central region in the rest of the paper, and the entire device behaves like a single-electron transistor. ${ }^{11,12}$

The aim of this work is to provide a theoretical background to understand how the magnetic state of localized magnetic moments affects transport through the graphene nanoconstriction in the SET regime. This is different from previous works where the influence of the magnetic state of magnetic edges ${ }^{13}$ and adsorbed hydrogens ${ }^{9}$ on the conductivity was studied in the ballistic regime, with a central island strongly coupled to the electrodes, and also in the diffusive regime ${ }^{14}$ as well as SET through graphene islands with magnetic zigzag edges. ${ }^{15}$

The paper is organized as follows. In Sec. II, we discuss a tight-binding Hamiltonian for the graphene island exchanged coupled to the spins of the magnetic adsorbates. The results of this microscopic calculation justify the use of a simple single-orbital spin-split model for the SET, discussed in Sec. III, together with the possible mechanisms that enable the magnetic sensing. Finally, conclusions are presented in Sec. IV.

\section{MODEL FOR GRAPHENE ISLAND WITH MAGNETIC ADSORBATES}

\section{A. Hamiltonian}

Our starting point is a microscopic model for electrons confined in a graphene nanoisland that are exchanged coupled to the magnetic centers. The graphene central island is described with a tight-binding Hamiltonian for the honeycomb lattice contained in a rectangular stripe of dimensions $L_{x} \times L_{y}$ [see Fig. 1(a)]. In order to avoid the spin-polarized states formed at the zigzag edges, ${ }^{16,17}$ we impose periodic boundary conditions in one direction so that the structure only has open edges of armchair type. The coupling to the magnetic moments of the adsorbate molecules $\vec{m}(i)$ is then assumed to be a local exchange $J$ or spin-dependent potential, affecting $N$ sites randomly selected in the graphene central island. For simplicity, we consider that the magnetic moments of the molecules are all oriented along the same axis, which we choose as the spin quantization axis. These assumptions are good approximations in the case of strongly uniaxial $\mathrm{TbPC}_{2}$ molecules. $^{2}$ Hence we can write the Hamiltonian of the graphene and adsorbates as

$$
\mathcal{H}=\mathcal{H}_{0}+J \sum_{i} m_{z}(i) S_{z}(i)+e V_{g}\left(N_{\text {TOT }}-\hat{N}\right),
$$

where $\mathcal{H}_{0}$ is the tight-binding Hamiltonian for $\pi$ electrons in graphene considering nearest-neighbor interactions, $J$ is the strength of the exchange coupling between the graphene electrons and the magnetic moment of the molecules, which can take two values, $m_{z}(i)= \pm 1$. Finally, the last term in the 


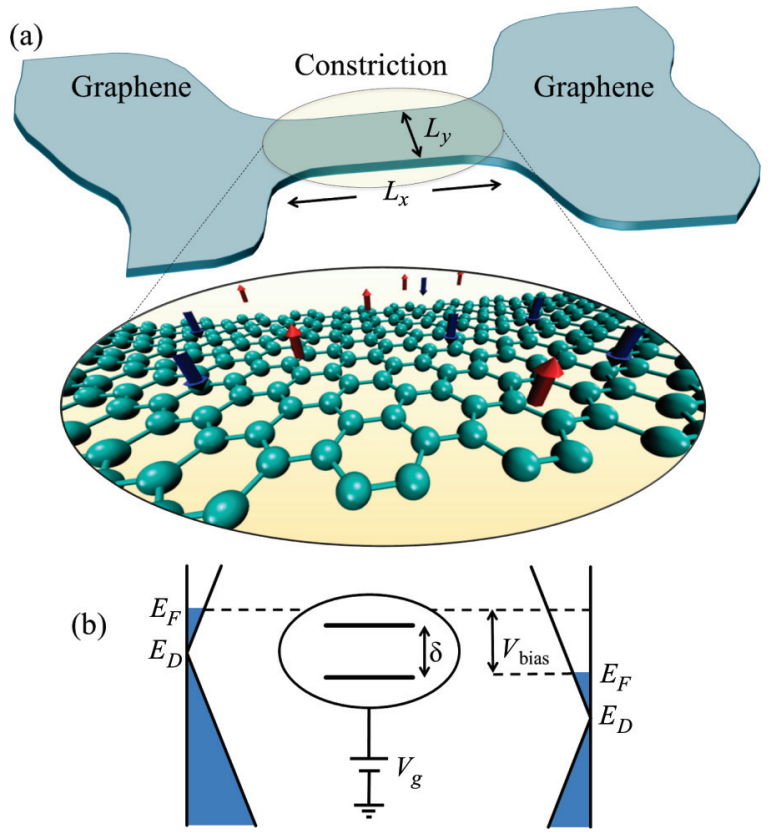

FIG. 1. (Color online) (a) Scheme of a graphene constriction with randomly distributed magnetic centers. (b) Diagram with the system energy levels and graphene density of states where $E_{D}$ is the energy of the Dirac point, $E_{F}$ is the Fermi level of the graphene electrodes, $V_{\text {bias }}$ is the bias voltage, $V_{g}$ is the gate voltage that controls the energy levels in the quantum dot and $\delta$ is the spin splitting of the transport level.

Hamiltonian describes the electrostatic coupling of the total charge of the dot, which can be either 0 or $e$, given by the difference in the number of electrons $\hat{N}$ and the number of carbon atoms $N_{\text {TOT }}$ in the central island. $S_{z}(i)$ is the local spin density of the $p_{z}$ electrons in graphene at site $i$,

$$
S_{z}(i)=\frac{1}{2}\left(c_{i \uparrow}^{\dagger} c_{i \uparrow}-c_{i \downarrow}^{\dagger} c_{i \downarrow}\right),
$$

where $c_{i \uparrow}^{\dagger}$ creates an $\uparrow$ electron at the $p_{z}$ orbital of site $i$ of graphene. In the following, we assume that magnetic fields controlling the spin orientation of the adsorbates are applied along the plane of graphene so that it is a good approximation to neglect the diamagnetic coupling to the graphene electrons.

There are several independent microscopic mechanisms for spin-dependent interaction between magnetic adsorbates and the graphene $\pi$ electrons that can be modeled with Eq. (1). In the case of magnetic molecule such as $\mathrm{TbPC}_{2}$, used in Ref. 2, the magnetic Tb atom is separated from the graphene electrons by the nonmagnetic atoms of the molecule, and the most likely mechanism for spin coupling is kinetic exchange. ${ }^{19}$ This coupling will generate a local Kondo-exchange between graphene electrons and the molecules. ${ }^{20}$ More complicated scenarios, like coupling of graphene electrons to unpaired electrons in the organic rings of the molecules, would imply that every molecule affects several sites in graphene. Direct dipolar coupling would also affect several sites per molecule, but the average magnetic field created by a magnetic moment of $5 \mu_{B}$ at $0.5 \mathrm{~nm}$ on a disk with an area around $400 \mathrm{~nm}^{2}$, the graphene constriction area in Ref. 2, is smaller than $1 \mu \mathrm{T}$, which would yield a negligible maximal Zeeman coupling of nano-electron-volts per molecule.
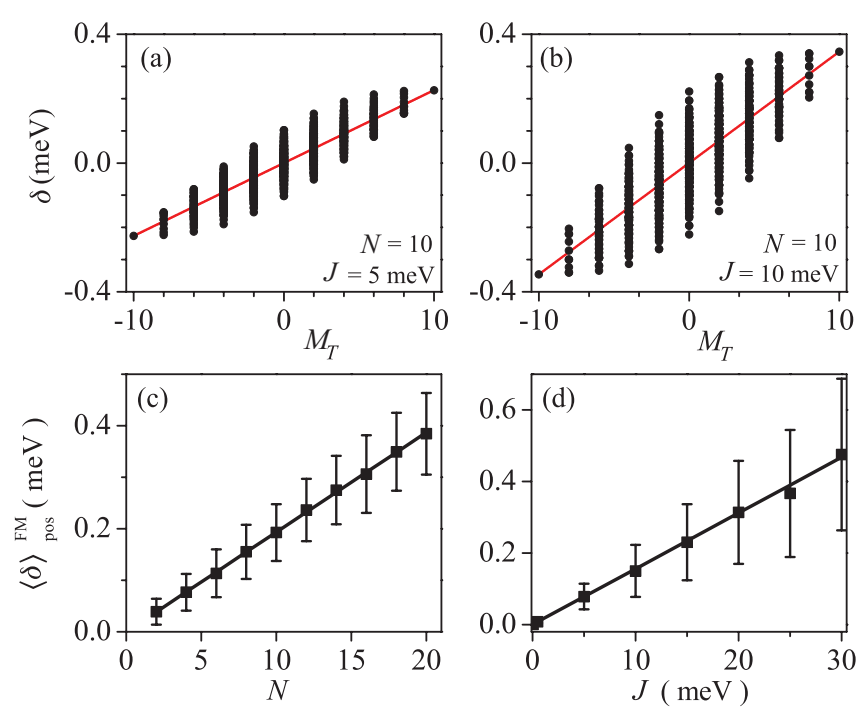

FIG. 2. (Color online) Spin splitting $\delta$ for a sample of dimensions $L_{y}=N_{y} a$ and $L_{x}=N_{x} \sqrt{3} a$, with $N_{x}=15$ and $N_{y}=17$, being $a=$ $2.46 \AA$ the lattice constant of graphene. $\delta$ vs total magnetization $M_{T}$ for a single spatial distribution of $N=10$ magnetic impurities with (a) $J=5$ and (b) $10 \mathrm{meV}$. The red solid line indicates the average value $\langle\delta\rangle_{\text {pos }}$. Average $\langle\delta\rangle_{\text {pos }}^{\mathrm{FM}}$ vs number of magnetic molecules $N$ for $J=5 \mathrm{meV}$ (c) and exchange energy $J$ for $N=4$ (d). The error bars correspond to the standard deviation.

\section{B. Relevant energy scales}

The reported dimensions of the central region, $L_{x} \simeq L_{y} \simeq$ $20 \mathrm{~nm}$, lead to an energy spacing $\Delta \epsilon$ of the single-particle spectrum much larger than the temperature and the charging energy. ${ }^{11,12} \mathrm{We}$ also assume that the exchange induced shifts are smaller than the single-particle splitting. As a result, the effect of exchange is to shift the bare energy levels, without mixing them. Thereby, we can safely assume that electrons tunnel through just one of the single-particle levels, which might be spin-split due to exchange with molecules, as is schematically shown in Fig. 1(b). We assume that the charge of the central island fluctuates between $q=0$ and $q=-|e|$, and that the transport level is the lowest unoccupied level of the central island spectrum. The energy of the transport level reads

$$
\epsilon_{T}^{\sigma}=\epsilon_{0}+\sigma \frac{\delta}{2}-|e| V_{g},
$$

where $\epsilon_{0}$ is the single-particle electron level, $\sigma= \pm 1$ denotes the spin direction and $\delta$ is the magnitude of the spin splitting, which is a functional of the magnetic landscape $\left\{m_{z}\right\}$.

Within our model, a given magnetic landscape is defined by the location $i$ and the magnetic state $m_{z}(i)$ of the $N$ magnetic adsorbates. In Figs. 2(a) and 2(b), we plot the value of $\delta$ for all the possible magnetic states of a given arrangement of $N=10$ atoms, for two different values of $J$. This choice corresponds to the estimated number of molecules in Ref. 2. These figures show a correlation between the magnitude of the splitting $\delta$ and the total magnetization $M_{T}$. The dispersion of $\delta$ for a fixed total magnetization $M_{T}$ is the outcome of indirect exchange coupling. ${ }^{18}$

For comparison with the experiments, it is worth considering two extreme magnetic landscapes. At large external field, all the magnetic moments are aligned, i.e., $m_{z}(i)=+1$. 
We refer to this as the ferromagnetic (FM) landscape. At magnetic fields smaller than the coercive field of the magnetic molecules, their average magnetization should be zero and thus, $\sum_{i} m_{z}(i)=0$. We refer to these cases as nonmagnetic (NM). In order to sample the positional disorder, we perform an average over positional configurations, both for NM and FM cases. For a fixed spin choice $\left\{m_{z}\right\}$ with $M_{T}=0$, an average over positional configurations yields $\langle\delta\rangle_{\text {pos }}=0$. The reciprocal statement is also true: for a fixed positional configuration, an average over all the magnetic landscapes with $M_{T}=0$ also yields an average $\langle\delta\rangle_{\text {spins }}=0$.

In Fig. 2, we plot the average $\langle\delta\rangle_{\text {pos }}^{\mathrm{FM}}-\langle\delta\rangle_{\text {pos }}^{\mathrm{NM}}=\langle\delta\rangle_{\text {pos }}^{\mathrm{FM}}$ over 500 realizations as a function of the number of molecules $N$ [see Fig. 2(c)] and as a function of the molecule electron exchange $J$ [see Fig. 2(d)]. We have also calculated $\langle\delta\rangle_{\text {pos }}^{\mathrm{FM}}$ fixing the number of magnetic centers $N$, the strength of the coupling $J$ and changing $N_{\text {TOT }}=N_{x} N_{y}$, the total number of carbon atoms in the island. We find that the results of all these simulations can be summarized in the following equation:

$$
\langle\delta\rangle_{\text {pos }}^{\mathrm{FM}} \approx \frac{N}{N_{\mathrm{TOT}}} J .
$$

Whereas this result has been obtained from exact numerical diagonalization of the Hamiltonian, this dependence can be rationalized using first-order perturbation theory, which yields the spin-dependent shift of the transport level:

$$
\Delta \epsilon_{T}^{\sigma}=\frac{\sigma}{2} J \sum_{i}^{N}\left|\phi_{T}(i)\right|^{2} m_{i},
$$

where $\phi_{T}(i)$ is the $J=0$ wave function of the transport orbital. We now use $\left|\phi_{T}(i)\right|^{2} \simeq \frac{1}{N_{\mathrm{TOT}}}$ so that we can approximate:

$$
\Delta \epsilon_{T}^{\sigma} \simeq \frac{\sigma}{2} \frac{J}{N_{\mathrm{TOT}}} M_{T} .
$$

Using the fact that $M_{T}=N$ for the FM configurations and 0 for the NM ones, we arrive to Eq. (4).

\section{SPIN-SPLIT SINGLE-ORBITAL MODEL FOR SET}

In this section, we discuss SET across a central island with a single spin-split particle level. This is justified by the results of the previous section. We obtain expressions for the current of the system and we discuss the conditions under which the conductance depends on the magnetic state of the single-electron transport.

\section{A. Single-electron transistor with a spin-split single-orbital model}

We consider single-electron transport though a spin-split single transport level, ${ }^{21}$ with energy $\epsilon_{T}^{\sigma}$. We assume that the occupation of the transport level can be either 0 or 1 , the doubly occupied configuration being much higher in energy. Within these approximations, the transport level has three relevant many-body states: uncharged and the two charged with $\uparrow$ or $\downarrow$ spins. In the zero-applied bias limit, each of these states will be occupied according to the thermal equilibrium distribution, which we denoted as $P_{0}, P_{\uparrow}$, and $P_{\downarrow}$, respectively. We are interested in the SET regime, and within the linear response $\left(e V_{\text {bias }} \ll k_{B} T\right)$, transport will be enabled only when the addition energy lies within the thermally broadened transport window defined by the applied bias.

Under these approximations, the current flowing from the left electrode to the central island is given by

$$
I=e \sum_{\sigma}\left(P_{0} W_{0 \rightarrow \sigma}^{L}-P_{\sigma} W_{\sigma \rightarrow 0}^{L}\right),
$$

where $W_{0 \rightarrow \sigma}^{L}$ and $W_{\sigma \rightarrow 0}^{L}$ are rates for electron tunneling from the left electrode to the dot and vice versa. Continuity equation ensures that this current is identical to the current flowing towards the right electrode and, thereby, equal to the net current flow. The tunneling rates for electron tunneling out of and into the $\operatorname{dot}^{22}$ are given respectively by

$$
W_{\sigma \rightarrow 0}^{L}=\frac{2 \pi}{\hbar}\left|T_{L}\right|^{2} \rho_{L}\left(\epsilon_{T}^{\sigma}\right)\left[1-f\left(\Delta_{\sigma}+V_{\text {bias }}\right)\right],
$$

and

$$
W_{0 \rightarrow \sigma}^{L}=\frac{2 \pi}{\hbar}\left|T_{L}\right|^{2} \rho_{L}\left(\epsilon_{T}^{\sigma}\right) f\left(\Delta_{\sigma}+V_{\text {bias }}\right),
$$

where $T_{L}$ is the strength of the dot-left electrode coupling, and

$$
\Delta_{\sigma} \equiv \epsilon_{T}^{\sigma}-E_{F},
$$

are the spin-dependent addition energies. Importantly, both $\delta$ and $V_{g}$ appear on equal footing, as additive quantities in this equation. The density of states of the left electrode, evaluated at the spin-dependent transport level energy, is denoted by $\rho_{L}\left(\epsilon_{T}^{\sigma}\right)$, while $f\left(\epsilon_{\sigma}\right)=\left(e^{\beta \Delta_{\sigma}}+1\right)^{-1}$ denotes the Fermi function. The electrode Fermi energy $E_{F}$ is taken to change linearly with the bias voltage $V_{\text {bias }}$. In the zero bias limit, the linear conductance reads

$$
G=G_{0} \sum_{\sigma}\left(P_{0}+P_{\sigma}\right) \frac{\Gamma_{\sigma}}{k_{B} T} \operatorname{Sech}^{2}\left(\frac{\beta \Delta_{\sigma}}{2}\right),
$$

where

$$
\Gamma_{\sigma} / \hbar=\frac{2 \pi}{\hbar}\left|T_{L}\right|^{2} \rho_{L}\left(\epsilon_{T}^{\sigma}\right),
$$

is the single-particle tunneling rate between the electrode and the transport level.

\section{B. Influence of magnetic state on conductance}

From the above discussion it is apparent that, for a given gate potential $V_{g}$ and temperature $T$, the linear conductance depends on the magnetic landscape affecting the central island through two classes of independent mechanisms, illustrated in Fig. 3: (i) the change of the addition energies $\Delta_{\sigma}$ which, as we show below, would result in a lateral shift of the $G\left(V_{g}\right)$ resonance curve [see Fig. 4(a)] and (ii) the change of the electron lifetime $\Gamma=\sum_{\sigma} \Gamma_{\sigma}$, that would result in a vertical resizing of the $G\left(V_{g}\right)$ resonance curve [see Fig. 5(a)].

In the first mechanism, the change in the magnetic state modifies the value of $\delta$, which must have a similar effect than changing the gate potential. It resembles the magnetoCoulomb effect, ${ }^{23,24}$ by which the applied magnetic field changes the Fermi energy of the electrode, shifting the $G\left(V_{g}\right)$ curves. However, this first mechanism necessarily implies a change of sign of the variation of $G$ as the gate potential is scanned along the resonance (see top panel of Fig. 4). Importantly, this is not observed in the experiments with 

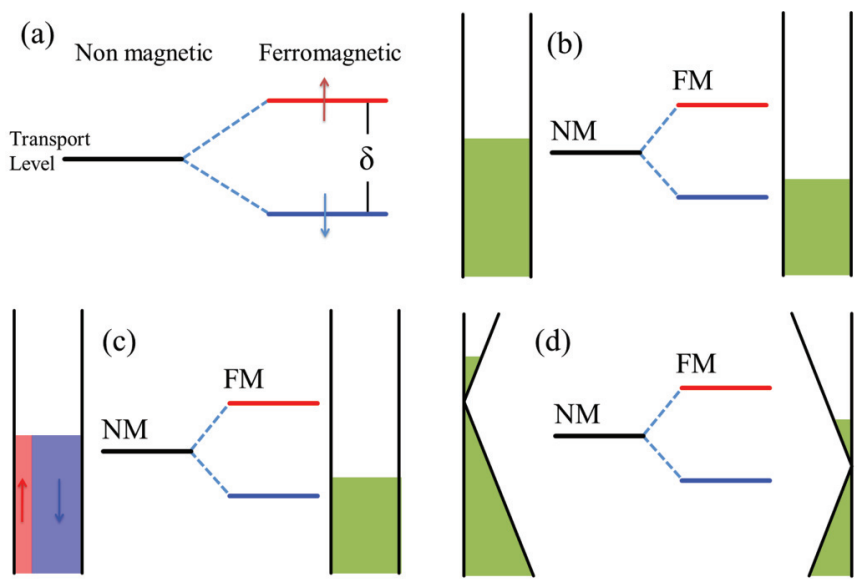

FIG. 3. (Color online) (a) Scheme showing the transport level energy splitting. Scheme of spin dependence of transport due to (b) detuning of the transport level with respect to the electrode Fermi energy, (c) magnetoresistance associated to spin polarized electrode(s), and (d) energy dependent tunneling rates.

magnetic molecules, ${ }^{2}$ but it is observed in the case of graphene nanoconstrictions. 5

Motivated by the behavior reported in Ref. 2, we pay attention also to the second mechanism. For spin unpolarized transport, the change in the transport energy level results in a change on tunneling rate $\Gamma / \hbar$ only if the electrode density of states depends on energy, which is exactly the case of graphene. For spin-polarized transport, the relative orientation of the electrode and island magnetic moments gives rise to magnetoresistive effects that are accounted for by the changes in $\Gamma_{\sigma}$

\section{Transport for constant tunneling rates}

We now discuss our transport simulations for the graphene single-electron transistor spin sensor. We focus on the first spin sensing mechanism in a single-electron transistor: changes in spin splitting of the transport level produce changes in addition energies $\Delta_{\sigma}$ [see Fig. 3(b)]. For that matter, we neglect both the energy and spin dependence of the tunneling rates $\Gamma_{\sigma}$. In Fig. 4(a), we show the linear conductance, in units of $g_{0}=$ $\beta \Gamma G_{0}$, as a function of gate voltage, for several values of the transport level splitting $\delta$, in units of $k_{B} T$. It is apparent that the Coulomb blockade peaks undergo a lateral shift, as expected from the fact that $V_{g}$ and $\delta$ appear on equal footing on the spin-dependent addition energies. At $\Delta=0$, the two spin channels contribute. Therefore, as we increase $\delta$, the height of the conductance peaks decreases, because one of the two spin channels is removed from the transport window of width $k_{B} T$.

In Fig. 4(b), we plot the variations in the linear conductances as a function of the spin splitting $\delta$, for several values of $V_{g}$. We see two types of curves. For values of $V_{g}$ such that the transport level is occupied, as we increase $\delta$, the transport level is pushed downwards, away from the elastic transport window, switching off the transistor conductance. In contrast, for $V_{g}$ such that the transport level is empty for $\delta=0$, lying above the elastic transport window, ramping $\delta$ makes one of the two spin states of the transport level enter the transport window, giving rise to the double-peak structure. The fact that $\delta$ and $V_{g}$ play analogous roles is illustrated in Figs. 4(c) and 4(d),
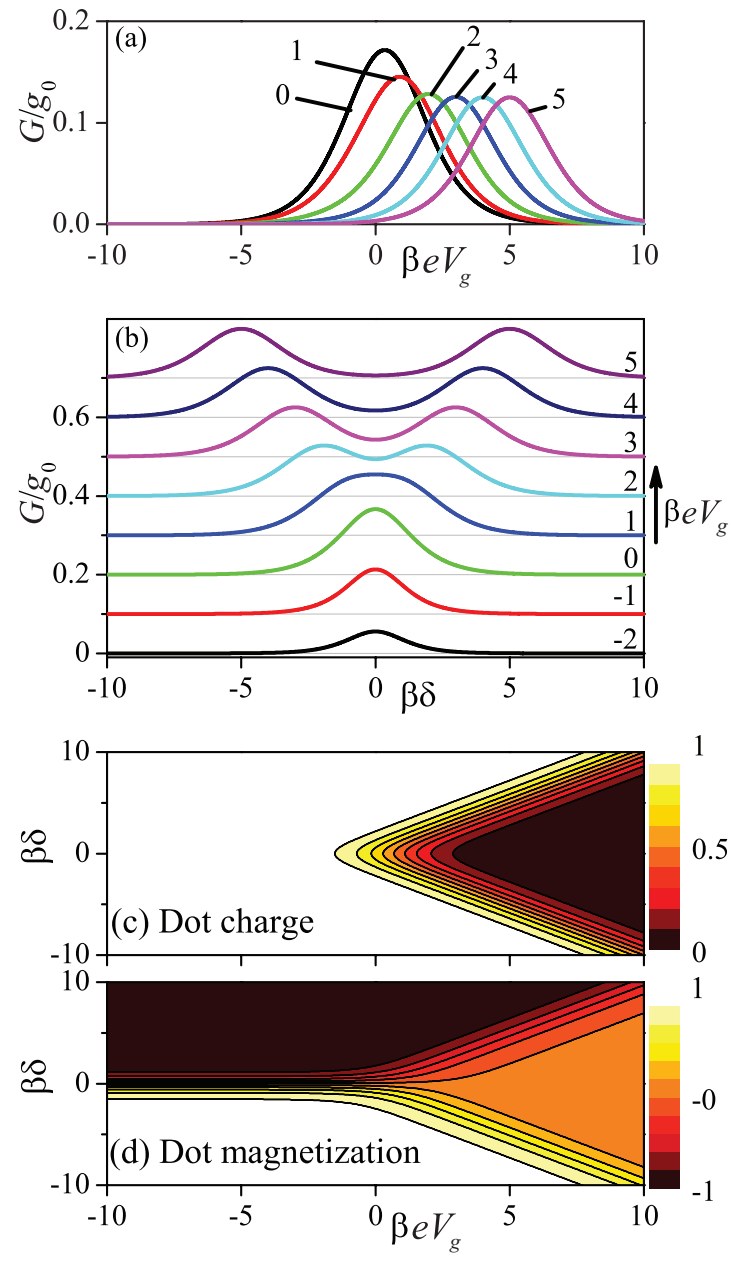

FIG. 4. (Color online) Conductance in units of $g_{0}=G_{0} \beta \Gamma$ as a function of the gate voltage $e V_{g}$ and the level splitting $\delta$. (a) The normalized conductance as a function of the gate potential $e \beta V_{g}$, where the labels corresponds to the different $\beta \delta$ values. (b) The conductance as a function of $\beta \delta$ for several values of the gate $e \beta V_{g}$. For the sake of clarity, all curves have been displaced by 0.1 . In (c) and (d), we present a contour plot of the dot charge (defined as $\left.Q=P_{\uparrow}+P_{\downarrow}\right)$ and the dot magnetization $\left(m=P_{\uparrow}-P_{\downarrow}\right)$ as a function of the gate voltage $e V_{g}$ and level splitting $\delta$.

where we show the average magnetization and occupation of the transport level in the phase diagram defined by these two variables.

\section{Transport with energy-dependent tunneling rates}

We now consider the second mechanism for spin sensing in a single-electron transistor: changes in spin splitting of the transport level produce changes in the tunneling rates $\Gamma_{\sigma}$. This can happen for the two following reasons. (1) One of the electrodes is spin polarized, so that $\Gamma_{\downarrow} \neq \Gamma_{\uparrow}$. Spin-polarized transport is sensitive to the product of the magnetic moment of electrode and central island. This type of effect has been thoroughly discussed in the case of SET with ferromagnetic electrodes. $^{25,26}$ (2) The density of states of the electrode depends on energy. Thus changes in the transport level change $\Gamma_{\sigma}$, for both spins. This is a natural scenario for graphene electrodes. ${ }^{32,33}$ 

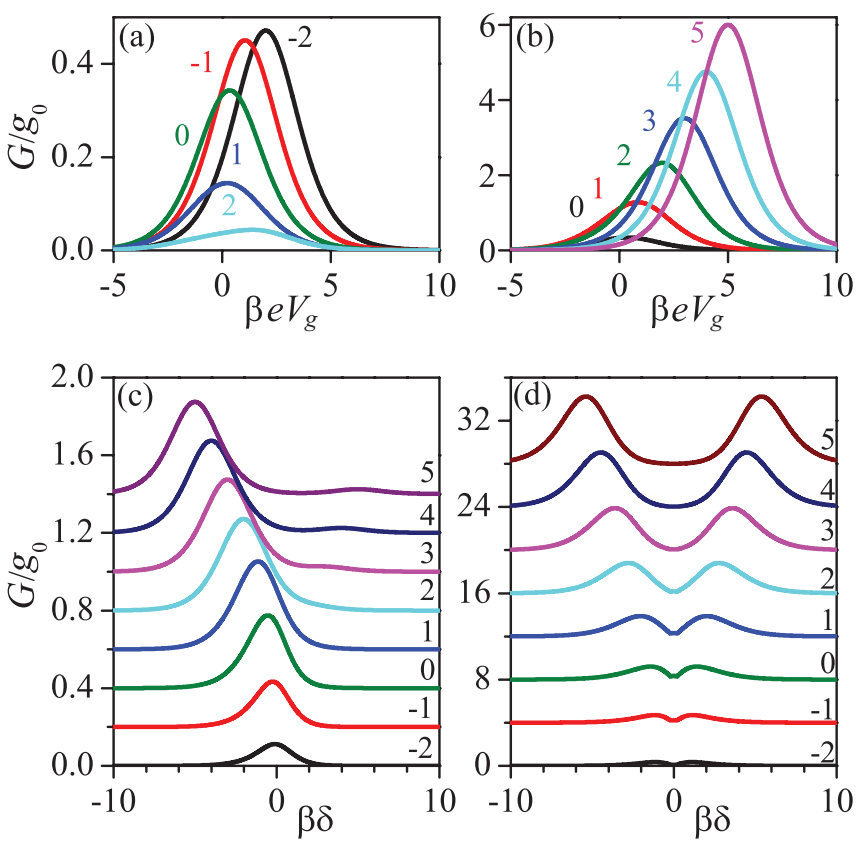

FIG. 5. (Color online) Normalized conductance for ferromagnetic electrodes as a function of the level splitting $\delta$ for several gate values. In (a) and (c), the density of states depends of the polarization $\rho=\rho_{0}(1+\sigma \mathcal{P})$, in this particular case, we take a polarization of $\mathcal{P}=0.9$. In (b) and (d), the electrode density of states is linear with the energy, $\rho\left(\Delta_{\sigma}\right)=\rho\left(\epsilon_{0}+\sigma \delta / 2-E_{D}\right)$. For the sake of clarity the conductance curves in (c) and (d) have been displaced by 0.2 and 4 units, respectively.

Let us consider first the case of spin polarized electrodes. We do the assumption that the density of states is spin dependent but energy independent: $\rho_{\sigma}=\rho_{0}(1+\sigma \mathcal{P})$, with $\mathcal{P}$ the electrode polarization. In Fig. 5(a), we plot the linear conductance versus $V_{g}$ curves for several values of the splitting $\delta$, assuming a large value of the electrode spin polarization $\mathcal{P}=0.9$. It is apparent that, on top of the shift of the resonance curve whose origin was discussed in the previous section, there is a change in the amplitude of the curve. Notice that $G\left(\delta=k_{B} T\right)$ and $G\left(\delta=2 k_{B} T\right)$ are smaller than $G(0)$ for all values of $V_{g}$. In this specific sense, the gate-independent spin contrast is similar to the experimental report with magnetic molecules. ${ }^{2}$ In Fig. 5(a), we show the linear conductance as a function of $\delta$ for different values of $V_{g}$. It is apparent that, as opposed to the case of nonmagnetic electrodes shown in Fig. 5(b), the function $G(\delta)$ is no longer an even functions, reflecting the magnetoresistive behavior. Basically, transport is favored when the spin polarizations of the electrode and the central island are parallel.

We now consider a non-spin-polarized electrode with an energy-dependent density of states. This scenario occurs naturally in graphene. If we consider idealized graphene electrodes, neglecting effects of interactions, disorder and confinement, we have $\rho(\epsilon)=\rho_{0}\left|\epsilon-E_{D}\right|$, where $E_{D}$ is the Dirac point. The $G\left(V_{g}\right)$ curves, shown in Fig. 5(b) for different values of $\delta$, shift and change amplitude. The shift is related to the change of the addition energies, discussed in the previous section, and the change in amplitude comes from the variation of the tunneling rate as the transport level scans the energy-dependent density of states of the electrode.

In Fig. 5(d), we plot $G(\delta)$ for several values of $V_{g}$. The curves are similar to the case with energy independent tunneling rates, except for the dip at zero $\delta$, which occurs because we chose the bare transport level right at Dirac point. This is the most favorable choice to maximize the effect of energy dependence of $\Gamma$. From our results, and given the fact that experimentally is not possible to put the Fermi energy arbitrarily close to the Diract point, ${ }^{28}$ we find it unlikely that this effect is playing a role in the experiments.

\section{E. Sensitivity of the single-electron transistor spin sensor}

We now discuss the sensitivity of the spin sensor based on the graphene single-electron transistor, as described by our model, neglecting changes in $\Gamma$. From Fig. 4(a), we propose, as rule of thumb, that variations of $\delta$ similar or larger than $k_{B} T$ can be resolved. Estimating $\delta$ from the case of fully spin-polarized magnetic adsorbates, given in Eq. (4), we find a relation between the minimal number of magnetic centers $N$ that can be detected, and the temperature and exchange constant:

$$
\frac{N}{N_{\text {TOT }}}>\frac{k_{B} T}{J} \text {. }
$$

It is apparent that decreasing the temperature, or increasing the spin-graphene exchange coupling, increases the sensitivity of the device (makes it possible to detect a smaller concentration of molecules). For instance, at $100 \mathrm{mK}$, and taking $N_{\text {TOT }} \sim 15000$, which corresponds to an approximate area of $400 \mathrm{~nm}^{2}$, one could detect 10 molecules for an exchange coupling $J \gtrsim 15 \mathrm{meV}$.

Recent reports have shown that it is possible to fabricate graphene nanoislands with lateral dimensions of $1 \mathrm{~nm} .{ }^{27}$ These dots have $N_{\text {TOт }}<100$. Thus they would permit the detection of the spin of a single magnetic adsorbate provided that $k_{B} T$ is kept hundred times smaller than $J$. For $T=100 \mathrm{mK}$, this implies $J>1 \mathrm{meV}$. Interestingly, in such a small dot Coulomb blockade persists even at room temperature, but increasing $k_{B} T$, keeping the sensitivity would require also to increase $J$.

\section{DISCUSSION AND CONCLUSIONS}

Because of its structural and electronic properties, graphene is optimal for a spin sensor device. Being all surface, the influence of adsorbates on transport should be larger than any other bulk material. Because of the linear relation momentum and large Fermi velocity, energy level spacing in graphene nanostructures can easily be larger than the temperature, the tunneling induced broadening, and the perturbations created by the adsorbates. One of the consequences is that single-electron transport takes place through a single-orbital level.

Our simulations show how the spin splitting $\delta$ of the transport level is sensitive to the average magnetization of the magnetic adsorbates, which is controlled by application of a magnetic field along the plane of graphene, to avoid diamagnetic shifts. On the other hand, the linear conductance $G$ of the single-electron transistor depends on $\delta$, which accounts for the sensing mechanism. More specifically, $G$ 
depends on $\delta$ due to either changes in the spin-dependent addition energies $\Delta_{\sigma}$ or changes in the electrons lifetime $\Gamma_{\sigma}$. The first is independent of the nature of the electrodes, whereas the second only happens if they are magnetic or have an energy-dependent density of states.

We have shown how, within an independent particle model and in the single-electron transport regime, the energy dependence of the graphene electrode density of states can only be relevant if the transport energy level is fine tuned to the Dirac point. However, this fine tuning is quite unlike to happen in experimental conditions. ${ }^{28}$ Still, the combined action of disorder and Coulomb interaction could give rise to a so-called Coulomb gap in the density of states of graphene, that might make the tunneling rates depend on the energy. ${ }^{29-31}$

Finally, we have assumed that both the edges of the graphene island and graphene electrodes are nonmagnetic. Our discussion of the effect of spin-polarized electrodes on the transport properties of the device would be valid for electrodes with ferromagnetic zigzag edges. A second possibility, out of the scope of this work, is to consider a graphene single-electron transistor whose central island has ferromagnetic edges. This case has been already studied. ${ }^{15}$

In conclusion, we have studied the mechanisms by which a graphene single-electron transistor could work as a sensor of the magnetic order of magnetic atoms or molecules adsorbed on the graphene central region. Our work has been motivated in part by recent experimental works. ${ }^{2,5}$ Whereas further work is still necessary to nail down the physical mechanisms for the spin sensing principles underlying the experimental work, our study provides a conceptual framework for graphene singleelectron transistor spin sensors.

\section{ACKNOWLEDGMENTS}

This work has been financially supported by MEC-Spain (Grant Nos. FIS2010-21883-C02-01 and CONSOLIDER CSD2007-0010) as well as Generalitat Valenciana, grant Prometeo 2012-11. We thank A. Candini for useful comments on the manuscript.
${ }^{1}$ F. Schedin, A. Geim, S. Morozov, E. Hill, P. Blake, M. Katsnelson, and K. Novoselov, Nat. Mater. 6, 652 (2007).

${ }^{2}$ A. Candini, S. Klyatskaya, M. Ruben, W. Wernsdorfer, and M. Affronte, Nano Lett. 11, 2634 (2011).

${ }^{3}$ S. Pisana, P. M. Braganca, E. E. Marinero, and B. A. Gurney, Nano Lett. 10, 341 (2010).

${ }^{4}$ D. Pesin and A. MacDonald, Nat. Mater. 11, 409 (2012).

${ }^{5}$ A. Candini, C. Alvino, W. Wernsdorfer, and M. Affronte, Phys. Rev. B 83, 121401 (2011).

${ }^{6}$ M. Urdampilleta, S. Klyatskaya, J. Cleuziou, M. Ruben, and W. Wernsdorfer, Nat. Mater. 10, 502 (2011).

${ }^{7}$ O. V. Yazyev and L. Helm, Phys. Rev. B 75, 125408 (2007).

${ }^{8}$ J. J. Palacios, J. Fernández-Rossier, and L. Brey, Phys. Rev. B 77, 195428 (2008).

${ }^{9}$ D. Soriano, F. Muñoz-Rojas, J. Fernández-Rossier, and J. J. Palacios, Phys. Rev. B 81, 165409 (2010).

${ }^{10}$ C. W. J. Beenakker, Phys. Rev. B 44, 1646 (1991).

${ }^{11}$ L. Ponomarenko, F. Schedin, M. Katsnelson, R. Yang, E. Hill,

K. Novoselov, and A. K. Geim, Science 320, 356 (2008).

${ }^{12}$ J. Güttinger, F. Molitor, C. Stampfer, S. Schnez, A. Jacobsen, S. Dröscher, T. Ihn, and K. Ensslin, Rep. Prog. Phys. 75, 126502 (2012).

${ }^{13}$ F. Muñoz-Rojas, J. Fernández-Rossier, and J. J. Palacios, Phys. Rev. Lett. 102, 136810 (2009).

${ }^{14}$ C. H. Lewenkopf, E. R. Mucciolo, and A. H. Castro Neto, Phys. Rev. B 77, 081410(R) (2008).

${ }^{15}$ M. Ezawa, Phys. Rev. B 77, 155411 (2008).

${ }^{16}$ J. Fernández-Rossier and J. J. Palacios, Phys. Rev. Lett. 99, 177204 (2007).

${ }^{17}$ O. V. Yazyev, Rep. Prog. Phys. 73, 056501 (2010).
${ }^{18}$ L. Brey, H. A. Fertig, and S. Das Sarma, Phys. Rev. Lett. 99, 116802 (2007).

${ }^{19}$ P. W. Anderson, Phys. Rev. 124, 41 (1961).

${ }^{20}$ J. R. Schrieffer and P. A. Wolff, Phys. Rev. 149, 491 (1966).

${ }^{21}$ P. Recher, E. V. Sukhorukov, and Daniel Loss, Phys. Rev. Lett. 85, 1962 (2000).

${ }^{22}$ H. Haug and A.-P. Jauho, Quantum Kinetics in Transport and Optics of Semiconductors (Springer-Verlag, Berlin, 1996).

${ }^{23}$ K. Ono, H. Shimada, and Y. Ootuka, J. Phys. Soc. Jpn. 66, 1261 (1997).

${ }^{24}$ S. J. Van Der Molen, N. Tombros, and B. J. Van Wees, Phys. Rev. B 73, 220406 (2006).

${ }^{25}$ J. Barnas, A. Fert Phys. Rev. Lett. 80, 1058 (1998).

${ }^{26}$ P. Seneor, A. Bernand-Mantel, and F. Petroff, J. Phys.: Condens. Matter 19, 165222 (2007).

${ }^{27}$ A. Barreiro, H. S. J. van der Zant, and L. M. K. Vandersypen, Nano Lett. 12, 6096 (2012).

${ }^{28}$ A. Mayorov, D. C. Elias, I. S. Mukhin, S. V. Morozov, L. Ponomarenko, K. S. Novoselov, A. K. Geim, and R. V. Gorbachev, Nano Lett. 12, 4629 (2012).

${ }^{29}$ S. Dröscher, H. Knowles, Y. Meir, K. Ensslin, and T. Ihn, Phys. Rev. B 84, 073405 (2011).

${ }^{30}$ A. L. Efros and B. I. Shklovskii, J. Phys. C: Solid State Phys. 8, 49 (1971).

${ }^{31}$ B. Terrés, J. Dauber, C. Volk, S. Trellenkamp, U. Wichmann, and C. Stampfer, Appl. Phys. Lett. 98, 032109 (2011).

${ }^{32}$ X. Liu, J. B. Oostinga, A. F. Morpurgo, and L. M. K. Vandersypen, Phys. Rev. B 80, 121407(R) (2009).

${ }^{33}$ F. Sols, F. Guinea, and A. H. Castro Neto, Phys. Rev. Lett. 99, 166803 (2007) 